\title{
Increasing the Dynamic Range of Synthetic Aperture Vector Flow Imaging
}

\author{
Villagómez Hoyos, Carlos Armando; Stuart, Matthias Bo; Jensen, Jørgen Arendt
}

\section{Published in:}

Proceedings of SPIE Medical Imaging 2014

Link to article, DOI:

$10.1117 / 12.2043637$

Publication date:

2014

Document Version

Early version, also known as pre-print

Link back to DTU Orbit

Citation (APA):

Villagómez Hoyos, C. A., Stuart, M. B., \& Jensen, J. A. (2014). Increasing the Dynamic Range of Synthetic Aperture Vector Flow Imaging. In Proceedings of SPIE Medical Imaging 2014 SPIE - International Society for Optical Engineering. https://doi.org/10.1117/12.2043637

\section{General rights}

Copyright and moral rights for the publications made accessible in the public portal are retained by the authors and/or other copyright owners and it is a condition of accessing publications that users recognise and abide by the legal requirements associated with these rights.

- Users may download and print one copy of any publication from the public portal for the purpose of private study or research.

- You may not further distribute the material or use it for any profit-making activity or commercial gain

- You may freely distribute the URL identifying the publication in the public portal 


\title{
Increasing the Dynamic Range of Synthetic Aperture Vector Flow Imaging
}

\author{
Carlos Villagomez Hoyos, Matthias Bo Stuart, \\ Jørgen Arendt Jensen \\ Center for Fast Ultrasound Imaging, Technical University of Denmark, 2800 Lyngby, Denmark
}

\begin{abstract}
In current ultrasound systems the dynamic range of detectable velocities is susceptible to the selected pulse repetition frequency, thus limiting the dynamic range of flow mapping. To establish the feasibility of extending the range of detectable velocities towards low velocity vessels, results are presented using synthetic aperture which increases the frame-to-frame signal correlation of the scatterer displacement while providing continuous data. In this paper, recursive synthetic aperture acquisition, directional beamforming, and cross-correlation are used to produce B-mode and vector velocity images. The emissions for the two imaging modes are interleaved 1-to-1 ratio, providing a high frame rate equal to the effective pulse repetition frequency of each imaging mode. The direction of the flow is estimated, and the velocity is then determined in that direction. This method works for all angles, including fully axial and transverse flows. The method is investigated using Field II simulations and data from the experimental ultrasound scanner SARUS, acquired from a circulating flow rig with a parabolic flow. A $7 \mathrm{MHz}$ linear array transducer is used, and several pulse repetition frequencies are synthesized in a simulated flow phantom with linearly increasing velocity and in a dual-vessel phantom with laminar flow with peak velocities of $0.05 \mathrm{~m} / \mathrm{s}$ and $0.5 \mathrm{~m} / \mathrm{s}$. The experimental measurements are made with laminar flow as in the simulations. For the simulated and experimental vessel with peak velocity of $0.05 \mathrm{~m} / \mathrm{s}$ and flow angle of $75^{\circ}$, the relative bias is $-0.29 \%$ and $-3.19 \%$, and the relative standard deviations are $2.39 \%$ and $5.75 \%$ respectively. For the simulated and experimental vessel with peak velocity of $0.5 \mathrm{~m} / \mathrm{s}$ and flow angle of $-90^{\circ}$, the relative biases are $-4.30 \%$ and $-7.37 \%$, and the relative standard deviations are $1.59 \%$ and $6.12 \%$, respectively. The presented method can improve the estimates by synthesizing a lower pulse repetition frequency, thereby increasing the dynamic range of the vector velocity imaging.
\end{abstract}

Keywords: vector flow imaging, high dynamic range, synthetic aperture

\section{INTRODUCTION}

Ultrasound can be used for blood flow imaging using a number of different approaches. Modern scanners utilize the autocorrelation estimator, ${ }^{1}$ which measures the phase shift as a function of time. Another approach is to use a time-shift based estimator, ${ }^{2}$ which determines the velocity by estimating the time-shift using the crosscorrelation function. Both estimators are traditionally used along the ultrasound beam direction, therefore they only find the velocity projected along this direction. Consequently blood flow imaging is highly susceptible to the beam-to-flow angle. Additionally, blood flow velocities varies in several orders of magnitude, e.g., pulsatile flow which in the carotid can vary from 0 to over $1 \mathrm{~m} / \mathrm{s}$ over the cardiac cycle.

In traditional ultrasound systems a trade-off is made between the range of detectable velocities and the number of estimation points. This can be overcome by using synthetic aperture for vector velocity estimation. ${ }^{3-6}$ Having continuous data available in all points enables estimation of velocities without compromising the pulse repetition frequency. In previous studies, Stuart et al ${ }^{7}$ estimated the vector velocity using synthetic aperture, but the high pulse repetition frequency hinders the angle estimation for slowly moving flow, hence providing incorrect estimates.

In this work, the approach suggested by Jensen, ${ }^{8}$ which merges the cross-correlation estimator with directional beamforming, is used in combination with a synthesized pulse repetition frequency. The basic principle is that as the data is continuously available at all estimation points, a lower pulse repetition frequency can be synthesized

Further information send correspondence to Carlos Villagomez Hoyos. E-mail: cavh@elektro.dtu.dk 
by simply skipping emissions. Then the direction of the flow can be estimated by cross-correlating time-shifted signals acquired in all directions. The direction of the flow is found where the correlation has its maximum, while the magnitude can be determined from the lag of the maximum correlation value.

The hypothesis investigated in this paper is that the method is not susceptible to the beam-to-flow angle and provides velocity estimates with a low standard deviation and an increased range.

This paper is organized as follows: Section 2 explains directional beamforming, recursive synthetic aperture, and presents the velocity estimation method, Section 3 describes the experimental setup for both simulations and measurements, Section 4 presents the results, and Section 5 gives discussion and conclusions.

\section{METHODS}

The following section describes the method for beamforming the directional signals and the approach taken for estimating vector velocities.

\subsection{Synthetic aperture}

The beamformation of the directional signals is performed as described by Jensen et al. ${ }^{9,10}$ The directional beamforming in synthetic aperture is obtained by focusing signals received by the transducer elements in a set of arbitrary points. These points can be located on a straight line in any direction.

The focusing of any of these points, is based on the total transmit and receive time-of-flight and a sample of the directional signal corresponding to the point $\mathbf{r}_{\mathbf{x}}$ is calculated as

$$
L_{i}\left(\mathbf{r}_{\mathbf{x}}\right)=\sum_{j=1}^{N_{E}} A_{i j}\left(\mathbf{r}_{\mathbf{x}}\right) s_{j}\left(\frac{\left|\mathbf{r}_{\mathbf{t}}-\mathbf{r}_{\mathbf{x}}\right|+\left|\mathbf{r}_{\mathbf{x}}-\mathbf{r}_{\mathbf{j}}\right|}{c}\right),
$$

where $N_{E}$ is the number of transducer elements, $A_{i j}\left(\mathbf{r}_{\mathbf{x}}\right)$ is the dynamic apodization coefficient, $s_{j}(t)$ is the received signal from transducer element $j, \mathbf{r}_{\mathbf{t}}$ is the position of the transmit source and $\mathbf{r}_{\mathbf{j}}$ is the position of the $j$ 'th transducer element.

To make a fully focused image, the beamformed signal $L_{i}\left(\mathbf{r}_{\mathbf{x}}\right)$ from each of the individual emissions are summed to give a high resolution image.

$$
g\left(\mathbf{r}_{\mathbf{x}}\right)=\sum_{i=1}^{N_{e}} L_{i}\left(\mathbf{r}_{\mathbf{x}}\right)
$$

where $N_{e}$ is the number of emissions. The image is constructed over a number of emissions, and if the emission sequence is repeating with no delay between the last emission of one iteration of the sequence and the first the first emission of the next iteration, data will be continuously available. In this case, recursive synthetic aperture (RSA) imaging is possible. ${ }^{11}$ In RSA imaging, the low-resolution image contribution from a given emission is replaced when that emission is repeated. In this way, new high-resolution images can be created at the rate of the pulse repetition frequency $(\mathrm{PRF})$. This makes it possible to average over more measurements when estimating velocities, and thus improves the standard deviation of the estimates.

RSA imaging can be applied to blood flow imaging when using cross-correlation estimators. ${ }^{12}$ For velocity estimation it is necessary to compare two measurements obtained in exactly the same way, so the difference is only the blood's movement between measurements. This implies that the time between the signals to be correlated is $T_{H R}$ seconds apart.

$$
T_{H R}=t_{p r f} \cdot N_{e},
$$

where $t_{p r f}$ is the time between emissions.

This paper combines RSA, directional beamforming, and synthetic aperture duplex imaging, which is a combination of two emission sequences in a 1-to-1 ratio to produce high-quality anatomical images and vector velocity estimates with high dynamic range and high frame rate. 


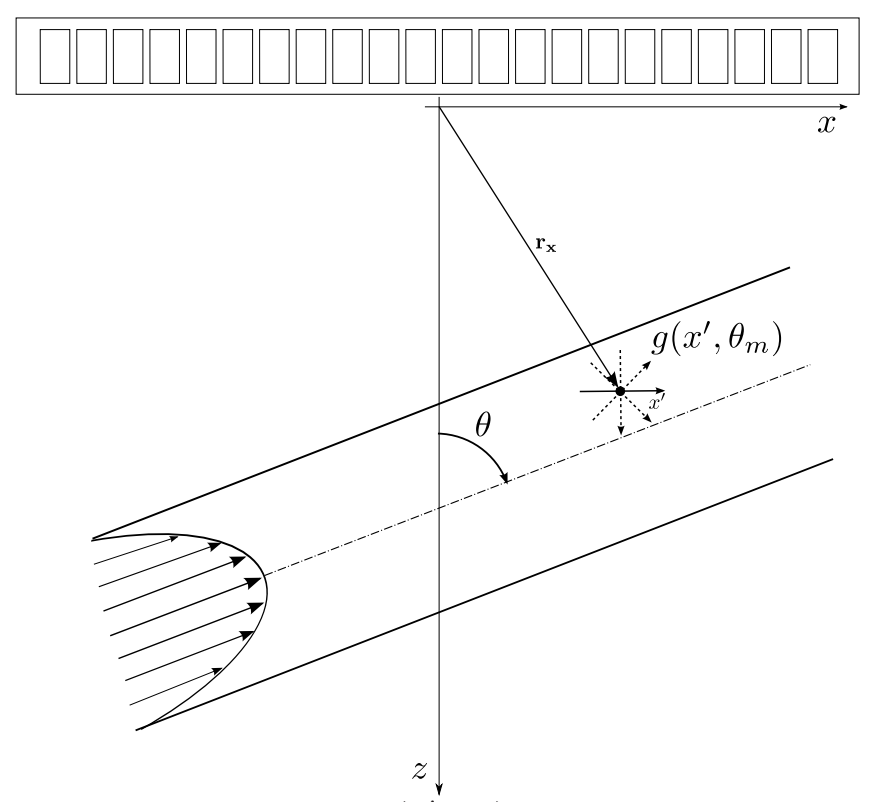

Figure 1: Directional signals $g\left(x^{\prime}, \theta_{m}\right)$ around estimation point $\mathbf{r}_{\mathbf{x}}$.

\subsection{Velocity vector estimation}

The angle and magnitude for the velocity vector estimation used in this paper are based on beamformed data generated by the RSA technique and directional beamforming, as explained in Section 2.1. The advantage of using SA compared to traditional beamforming in flow estimation is that SA produces continuous data for all estimation points. Therefore the number of samples for velocity estimation is only restricted by the time which the flow can be assumed quasi-stationary. In SA, lines can be focused in any direction, thus beamforming lines along the direction of the flow is possible, therefore estimating the true velocity magnitude using the crosscorrelation approach. Directional signals $g_{1}\left(x^{\prime}, \theta_{m}\right)$ and $g_{2}\left(x^{\prime}, \theta_{m}\right)$ are obtained at angle $\theta_{m}$ as illustrated in Fig. 1 . Here the $x^{\prime}$-axis is parallel to the $\theta_{m}$ angle direction and centred at a given estimation point $\mathbf{r}_{\mathbf{x}}$, and the signals are $T_{c c}$ seconds apart:

$$
T_{c c}=k_{t p r f} \cdot T_{H R},
$$

where $T_{H R}$ is the time between high resolution lines made with the same emission sequence, as defined in (3), and $k_{t p r f}$ indicates the number of frames to skip in the correlation. Cross-correlating the discrete version of the signals gives

$$
R_{12}\left(l, \theta_{m}\right)=\sum_{n=-N_{x c o r r} / 2}^{N_{x c o r r} / 2} g_{1}\left(n, \theta_{m}\right) g_{2}\left(n+l, \theta_{m}\right),
$$

where $l$ is the correlation lag and $N_{\text {xcorr }}$ is the discrete length of the directional signals.

To have a correct estimate of the velocity magnitude, the directionally beamformed line should match the direction of the flow. In general, this direction is not known and can be time dependent for non-constant flow.

The direction of the flow is found by cross-correlating along lines with different angles centered on the estimation point $\mathbf{r}_{\mathbf{x}}$. The true direction is the one that has the highest peak in the cross-correlation given that the signals decorrelate slower in the direction of the flow. This can be stated as:

$$
\begin{aligned}
R_{12}\left(\theta_{m}\right) & =\frac{\max \left(R_{12}\left(l, \theta_{m}\right)\right)}{\sqrt{R_{11}\left(0, \theta_{m}\right) R_{22}\left(0, \theta_{m}\right)}} \\
\theta & =\underset{\operatorname{Arg} \max R_{12}\left(\theta_{m}\right),}{\theta_{m}}
\end{aligned}
$$


where $\max \left(R_{12}\left(l, \theta_{m}\right)\right)$ is the maximum value of the cross-correlation function across angles $\theta_{m}$, and $R_{11}\left(0, \theta_{m}\right)$ and $R_{22}\left(0, \theta_{m}\right)$ are the corresponding power of the cross-correlated signals. The correct angle $\theta$ is found where the normalized correlation function as a function of angle, $R_{12}\left(\theta_{m}\right)$, has its peak value.

Once the direction of the flow is known, $g_{2}(n, \theta)$ can be considered as $g_{1}(n, \theta)$ time-shifted, and therefore the lag where the cross-correlation function has a maximum

$$
l_{\max }=\underset{l}{\arg \max }\left\{R_{12}(l, \theta)\right\},
$$

is, thus, the discrete shift in spatial position of the scatterers over the time interval $T_{c c}$. For increased accuracy, a second order polynomial is fitted to the cross-correlation around $l_{\max }$, and the position of the maximum correlation, $\hat{l}_{\max }$, is found by the interpolation formula ${ }^{13}$

$$
\hat{l}_{\max }=l_{\max }-\frac{R_{12}\left(l_{\max }+1, \theta\right)-R_{12}\left(l_{\max }-1, \theta\right)}{2 R_{12}\left(l_{\max }+1, \theta\right)-2 R_{12}\left(l_{\max }, \theta\right)+R_{12}\left(l_{\max }-1, \theta\right)} .
$$

The velocity along the flow direction is then given by

$$
\hat{v}_{x^{\prime}}=\frac{\hat{l}_{\max } \cdot d_{r}}{T_{c c}}
$$

where $d_{r}$ is the spatial sampling interval.

The probability of false peak detection is reduced by averaging over several estimates of $R_{12}$ using the assumption that the velocity of the scatterers can be considered constant for several pulses. A number of cross-correlations, $N_{x c}$ can, thus, be averaged in the estimation.

The maximum detectable velocity is limited by the search range $l_{\text {range }}$ and the minimum time interval $T_{c c}$, which corresponds to a $k_{\text {tprf }}$ of 1 ,

$$
v_{\text {max }}=\left(l_{\text {range }} \cdot d_{r}\right) / T_{H R},
$$

The size of the search range which must be chosen carefully as the use of a large search range may increase the probability of false peak detection. ${ }^{14}$

At the other end, the minimum detectable velocity is dependent on the echo canceling filter, performed by subtracting the average of a moving window of $N_{e c}$ high resolution lines. The minimum detectable velocity can be roughly estimated using the cut-off frequency of the stationary echo canceling filter and the distance between samples.

$$
v_{\min } \approx \frac{d_{r}}{T_{c c} \cdot N_{e c}}
$$

where $N_{e c}$ is the number of emissions used in the filter.

The parameters used in estimating the flow are listed in Table 1.

Table 1: Flow estimation parameters

\begin{tabular}{lll}
\hline \hline Parameter & Symbol & Value \\
\hline Spatial sampling interval & $d r$ & $0.1 \lambda$ \\
Spatial extent of directional signals & $X_{c o r r}$ & $-12.5 \lambda: 12.5 \lambda$ \\
Number of correlations for each estimate & $N_{x c}$ & 15 \\
Angular distance between directional lines & $d \theta_{m}$ & 5 \\
HR lines frequency & $1 / T_{H R}$ & $625 \mathrm{~Hz}$ \\
Correlation time factor & $k_{t p r f}$ & $1,2,4,6$ \\
Number of HR lines used for echo canceling & $N_{e c}$ & 16 \\
\hline \hline
\end{tabular}


Table 2: Default parameters used in all simulations.

\begin{tabular}{ll}
\hline \hline Parameter & Value \\
\hline Number of transmit elements & 64 \\
Number of receive elements & 128 \\
Transducer center frequency & $7 \mathrm{MHz}$ \\
Pitch of transducer element & $0.208 \mathrm{~mm}$ \\
Height of transducer element & $4.5 \mathrm{~mm}$ \\
Kerf & $0.035 \mathrm{~mm}$ \\
Speed of sound & $1480 \mathrm{~m} / \mathrm{s}$ \\
Transmit apodization & Hanning \\
Receive apodization & Rectangular (Boxcar) \\
Excitation Pulse & 2 period sinusoid \\
Pulse repetition frequency & $10 \mathrm{kHz}$ (Duplex) \\
RF sampling frequency & $100 \mathrm{MHz}$ \\
Virtual emission focus (flow) & Linear sweep at depth of -80 mm \\
Emissions per high resolution line (flow) & 8 \\
Number of frames & 150 \\
\hline \hline
\end{tabular}

\section{EXPERIMENTAL SETUP}

This section describes the simulations and measurements used for validating the method. First, the performance of the method is assessed for different conditions using simulations showing the capability of the method to extend the range of detectable velocities with the same data-set. Second a dual vessel simulation is done for evaluating the method during clinical conditions. Finally measurements are setup to match the dual-vessel simulation.

\subsection{Field II Simulations}

Field $\mathrm{II}^{15,16}$ is used to evaluate the performance of the estimator under different conditions. The velocity and angle estimates are obtained from simulated data without any added noise. A $7 \mathrm{MHz}, 192$ element, $\lambda$-pitch, linear array transducer is used with a sequence of eight defocused emissions for flow, interleaved with a standard synthetic aperture B-mode sequence. A 2-cycle sinusoidal pulse with a center frequency of $7 \mathrm{MHz}$ is used for both B-mode and flow. The parameter values for the simulations are listed in Table 2.

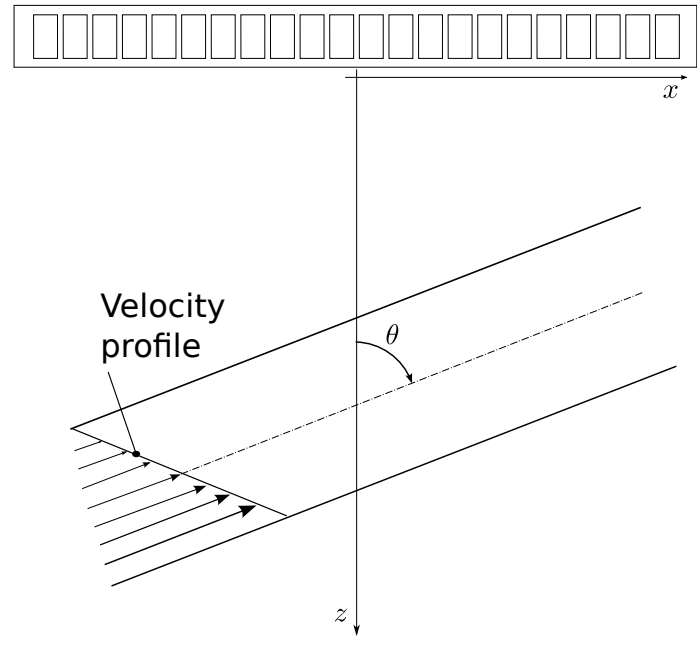

(a)

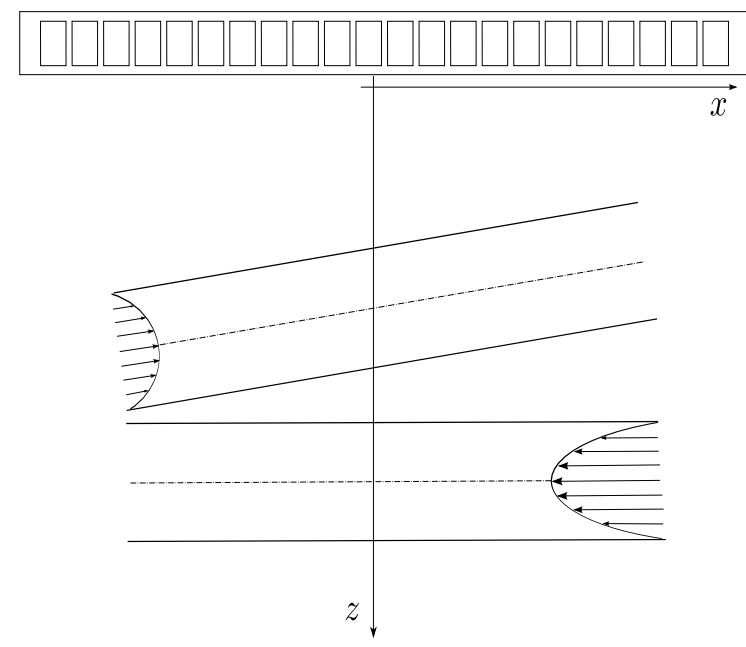

(b)

Figure 2: Phantoms used in simulations. (a) Gradient phantom. (b) Dual-vessel phantom. 


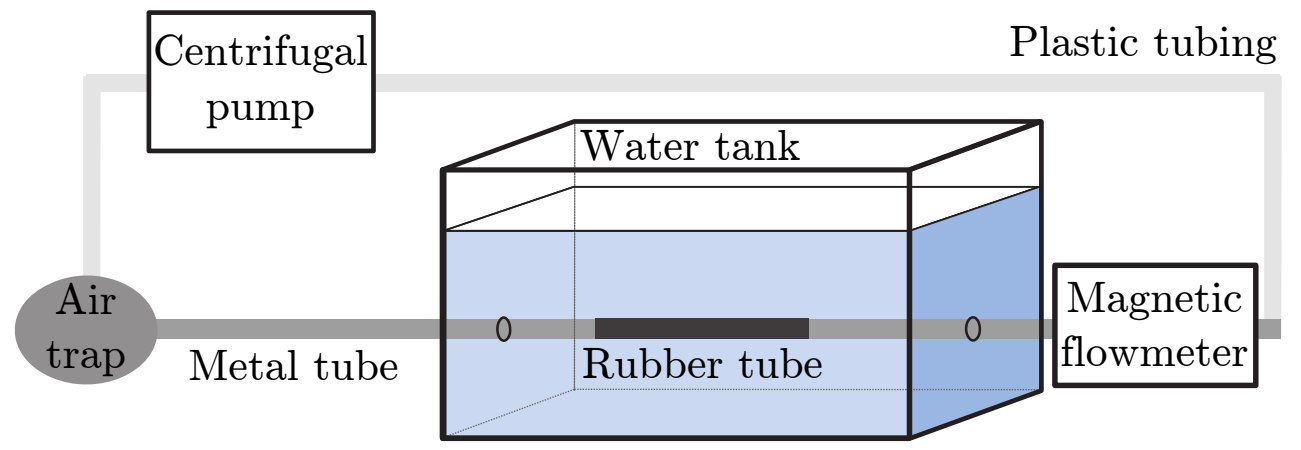

Figure 3: Illustration of the flow-rig system. It consists of a centrifugal pump, an air trap, a long rigid metal tube extending into a water tank followed by a magnetic flow meter. A transducer fixation device can be lowered into the water tank at adjustable beam-to-flow angles.

\subsubsection{Gradient phantom}

In order to evaluate the performance of the estimator at different velocities and angles, a gradient phantom is designed. Specifically, the phantom consists of blood mimicking scatterers moving with a Couette flow profile, see Fig. 2 (a). The velocity varies from $0 \mathrm{~cm} / \mathrm{s}$ at one vessel wall to $50 \mathrm{~cm} / \mathrm{s}$ at the other vessel wall. This flow is simulated at different angles $\theta=\{0,30,60,90\}$. The size of the simulated phantom is $2 \times 2 \mathrm{~cm}$, which includes stationary scatterers using 10 scatterers per resolution cell for fully-developed speckle. The vessel radius is $1 \mathrm{~cm}$.

The velocity and direction are estimated at $0.2 \mathrm{~mm}$ intervals along a line orthogonal to the vessel to cover the same velocity profile. Estimates are done for different $k_{t p r f}=\{1,2,4,6\}$, and the performance of the estimator is evaluated.

To evaluate the performance, the ratio between correct estimates and the total number of estimates is used. An estimate is considered acceptable when the estimated angle is within $\pm 5^{\circ}$ of the true angle and a maximum $20 \%$ error from the true velocity is estimated. The number of estimates is $\{1072,944,688,432\}$ for each $k_{t p r f}$, respectively.

\subsubsection{Dual-vessel phantom}

Parabolic flow profiles are simulated using Field II. The simulated vessels have a diameter of $10 \mathrm{~mm}$, are centred at 25 and $43 \mathrm{~mm}$ and have an angle of $75^{\circ}$ and $90^{\circ}$, respectively as seen in Fig. 2 (b). The peak velocities simulated for each parabolic flow profile are $0.05 \mathrm{~m} / \mathrm{s}$ for the top vessel and $0.50 \mathrm{~m} / \mathrm{s}$ for the lower vessel. The direction of the flow is in the positive $\mathrm{x}$-axis direction for the top vessel and negative $\mathrm{x}$-axis direction for the lower vessel.

\subsection{Measurement setup}

The experimental ultrasound scanner, SARUS, ${ }^{17}$ and a $7 \mathrm{MHz}$ linear array transducer, type 8804 (BK Medical, Herlev, Denmark), are used for all measurements. The system acquires RF data from the individual transducer channels, and these data are transferred to a PC, where they are saved and processed off-line. The received RF data contains 192 channels sampled at $35 \mathrm{MHz}$ and has 12 bits resolution for each individual channel.

All measurements are made in a flow-rig system as illustrated in Figure 3. A Cole-Parmer centrifugal pump (Vernon Hills, IL, USA) circulates a blood-mimicking fluid ${ }^{18}$ (Danish Phantom Design, Frederikssund, Denmark) in the closed loop circuit. The vessel radius is $6 \mathrm{~mm}$, and length long enough to ensure fully-developed laminar flow with a parabolic profile. The volume flow rate is measured by a calibrated MAG1100 flowmeter (Danfoss, Nordborg, Denmark) and used for calculating the peak velocity based on the expected parabolic profile. In its concentrated form, the fluid contains $5 \mu \mathrm{m}$-sized orgasol particles dissolved in glycerol, detergent, and demineralized water. For use, it is diluted in a ratio 1:20 with demineralized water, and dextran is added to obtain a viscosity $\mu$ of $3.9 \mathrm{mPa} \cdot \mathrm{s}$. The density $\rho$ is $1.0 \times 10^{3} \mathrm{~kg} / \mathrm{m}^{3}$. The peak velocity can be set based on the flow rate. The flow rate was adjusted to obtain a peak velocity of approximately 0.05 and $0.5 \mathrm{~m} / \mathrm{s}$. 
Inside the water tank a fixture for the transducer is inserted. The angle of the transducer and the distance to the tube are adjusted to match the geometry of the simulation setup. Two measurements are obtained, one for each simulated vessel.

\section{RESULTS}

The angle and velocity are estimated for simulations and measurements as described in Section 2.2. For all the results, the bias and standard deviation are calculated from the true angle, and the relative bias and relative standard deviation are calculated from the true velocity profiles. For both simulated and measured vessels, the velocity profile used as reference is a parabolic laminar flow described by;

$$
v(r)=v_{0}\left(1-\left(\frac{r}{R}\right)^{2}\right)
$$

where $R$ is the tube radius, $v_{0}$ is the maximum velocity at the center of the tube, and $r$ is the radial distance from the center of the tube.

All data are then processed off-line using a beamformation toolbox developed in-house (BFT3). ${ }^{19}$

\subsection{Simulations results}

\subsubsection{Gradient phantom}

The influence of the variation of $k_{t p r f}$ on the method is evaluated. As the method estimates the direction first and then the velocity, a wrongly detected direction most likely leads to an erroneous velocity estimate. For this reason, the estimates are split in two groups. A group of correct estimates, which are these that fall within a $\pm 5^{\circ}$ and $\pm 20 \%$ of the true direction and velocity, and a second group that are those outside this region. The performance of the estimator is then defined as the ratio of the number of correct estimates to the total number of estimates.

The angle bias and standard deviation are calculated for all the estimates, but the relative velocity bias and relative velocity standard deviation are solely based on the estimates that fall within $\pm 5^{\circ}$ of the true direction. This consideration is based on the influence of the outliers on the relative bias and relative standard deviation which therefore do not represent the performance properly.

As seen in Fig. 4, for a $k_{t p r f}$ of 2 , a range of velocities can be estimated accurately with a low standard deviation and bias, in both angle an magnitude, for any given angle. When the $k_{\text {tprf }}$ is changed, as seen in Fig. 4 (b), the range of detected velocities is shifted towards the lower speeds. This is explained by the increased $T_{c c}$ causing fast moving scatterers to move further and thereby decorrelate between observations. Similarly, slow moving scatterers are better detected since the echo canceling filter is also shifted.

\subsubsection{Dual-vessel phantom}

Fig. 5 shows the mean and standard deviation of the velocity profiles using different $k_{t p r f}$. The velocity vector is accurately determined without previous knowledge of the flow direction. However, for the vessel with $v_{\text {peak }}=0.05$ $\mathrm{m} / \mathrm{s}$, a higher $k_{t p r f}=6$ is needed to keep the standard deviation low. This can be achieved by taking advantage of the continuous existence of data in synthetic aperture, therefore removing any theoretical limit on the lower limit of the detectable velocities.

In Table 3 the relative bias and relative standard deviation of the simulated vessels is shown for different $k_{t p r f}$. It can be seen that when increasing $k_{\text {tprf }}$ the relative standard deviation for the vessel with $v_{\text {peak }}=0.05$ $\mathrm{m} / \mathrm{s}$ is improved, but for the vessel with $v_{\text {peak }}=0.5 \mathrm{~m} / \mathrm{s}$ the standard deviation is worsened. This happens because as more time passes the blood scatterers move out the beamformed line, and the signal decorrelates. 


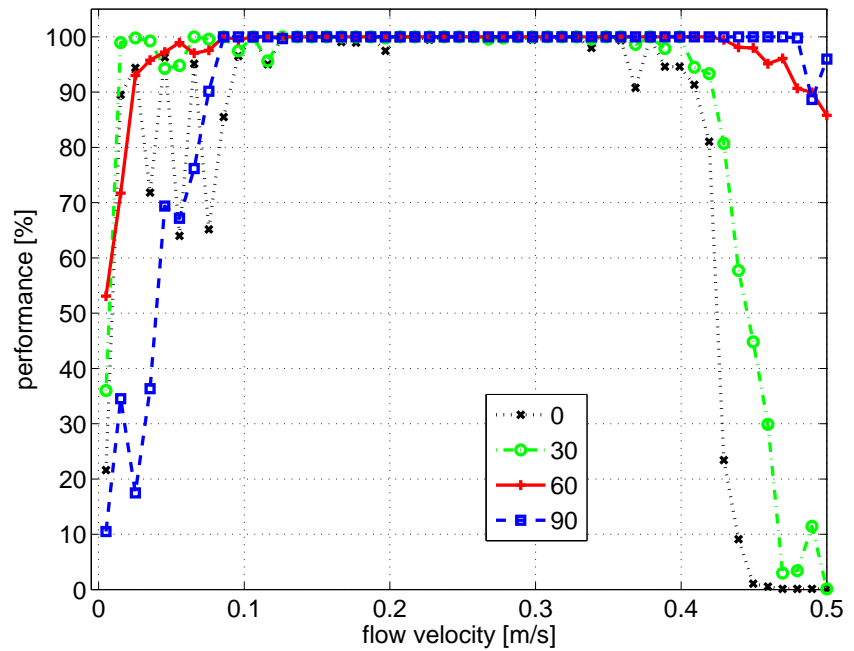

(a)
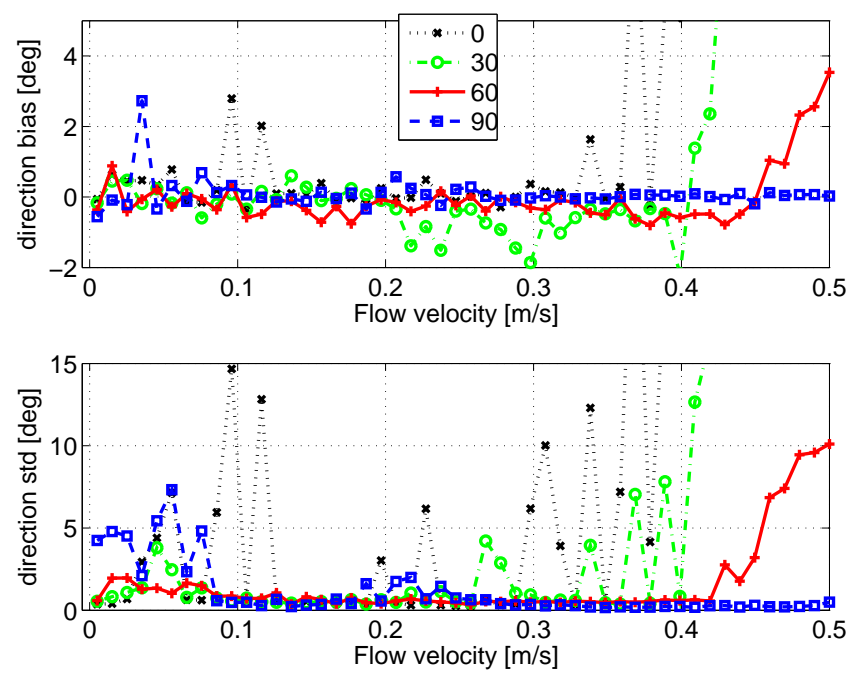

(c)

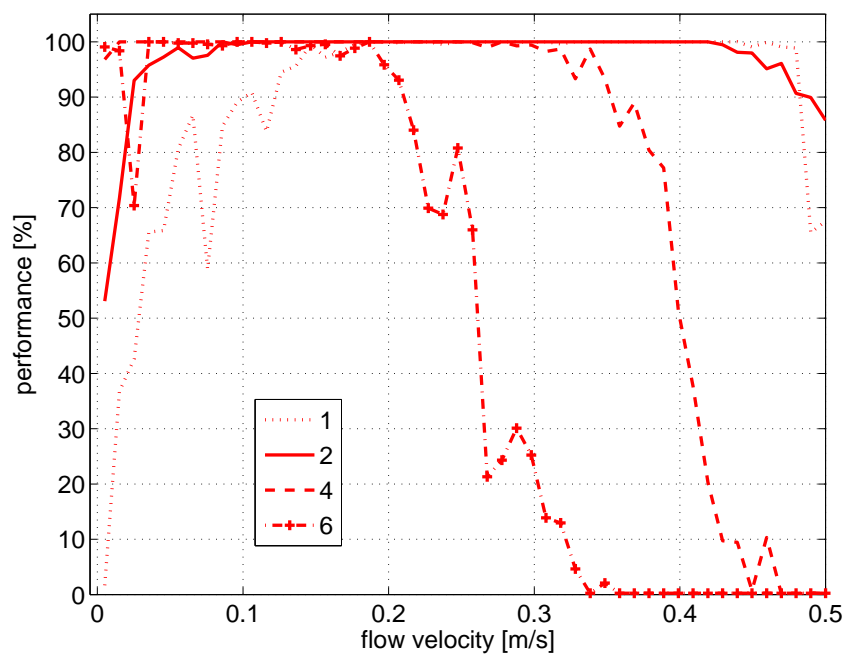

(b)
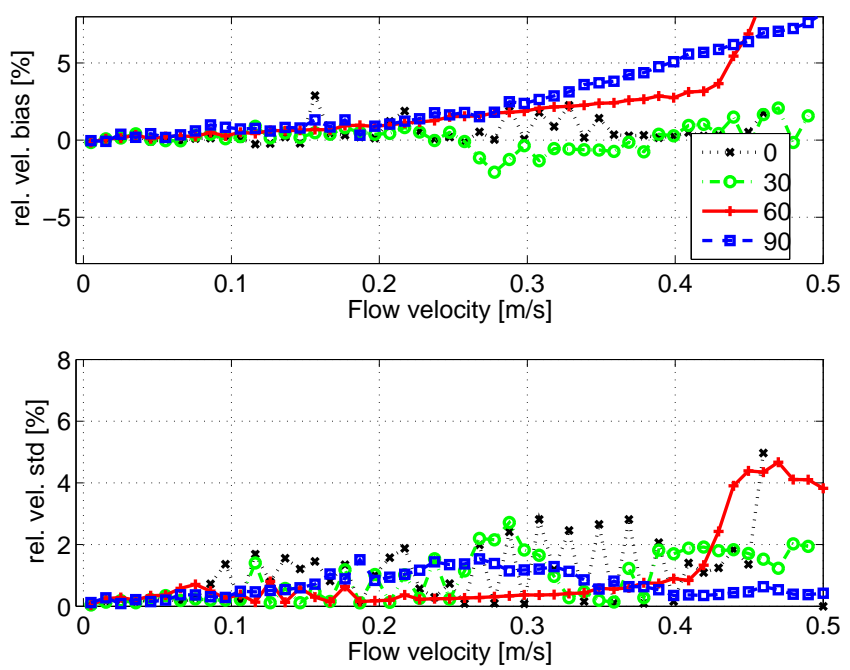

(d)

Figure 4: Performance of the estimation as a function of true velocity. (a)Percentage of correct estimates, i.e, estimates within $\pm 5^{\circ}$ and $20 \%$ of the true velocity vector for $\theta=0^{\circ}, 30^{\circ}, 60^{\circ}, 90^{\circ}$ at $k_{\text {tprf }}$ of 2 . (b)Percentage of correct estimate for $\theta=60^{\circ}$ and $k_{t p r f}=1,2,4,6$. (c)Bias and standard deviation for the angle at $k_{t p r f}$ of 2 . (d)Relative bias and relative standard deviation for the velocity magnitude for the subset of estimates that are within $\pm 5^{\circ}$ of the true direction at $k_{t p r f}$ of 2 . 


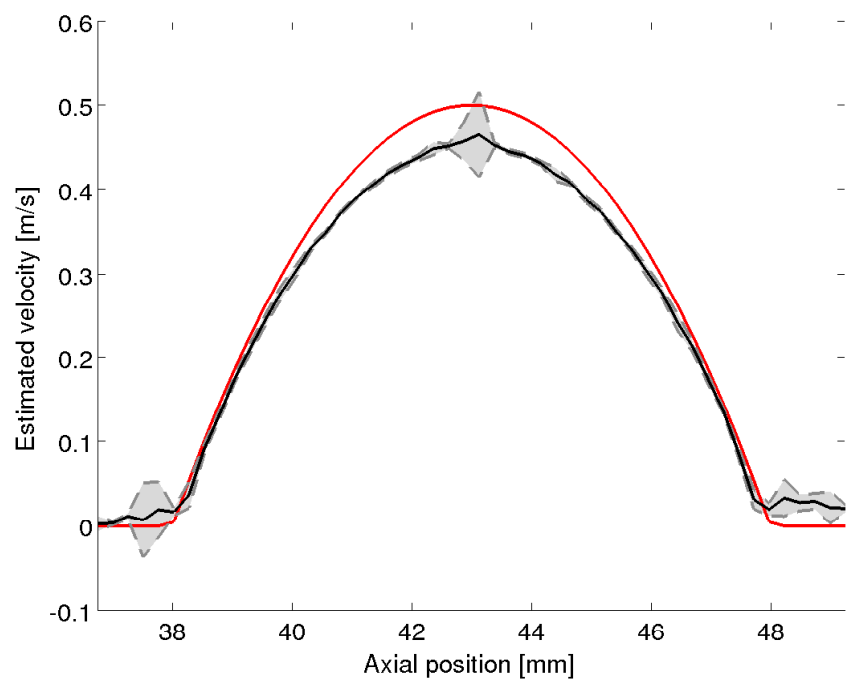

(a)

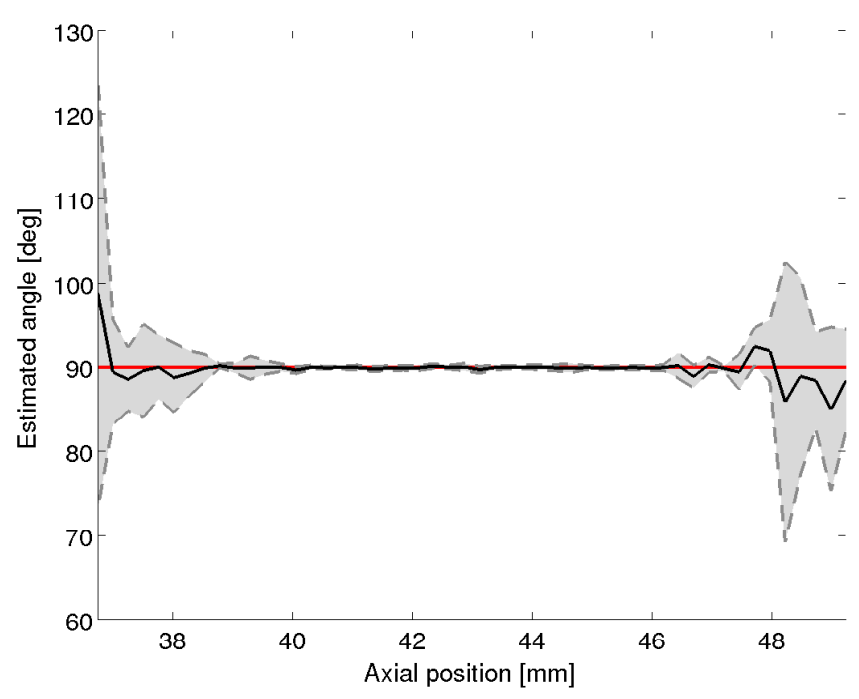

(c)

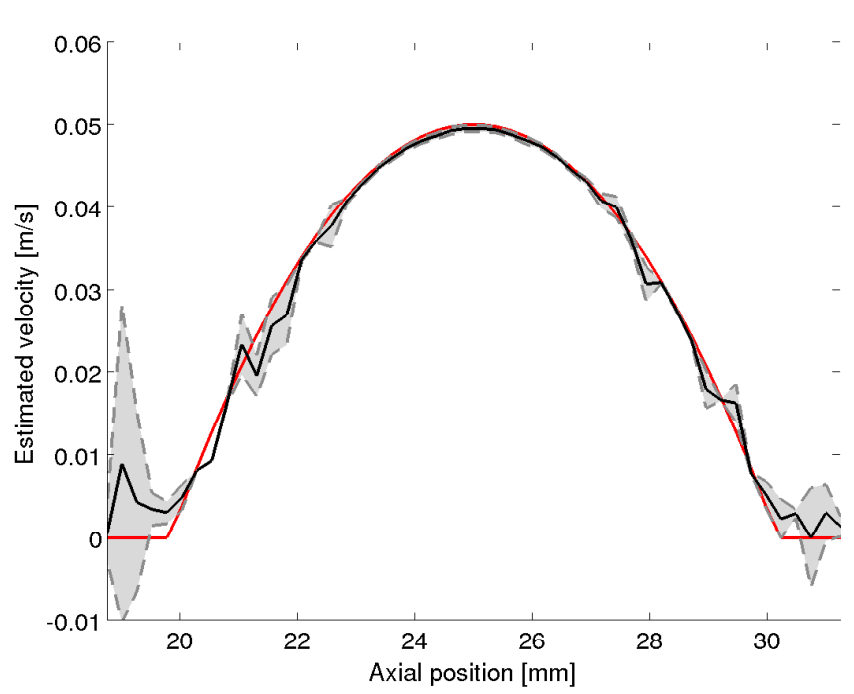

(b)

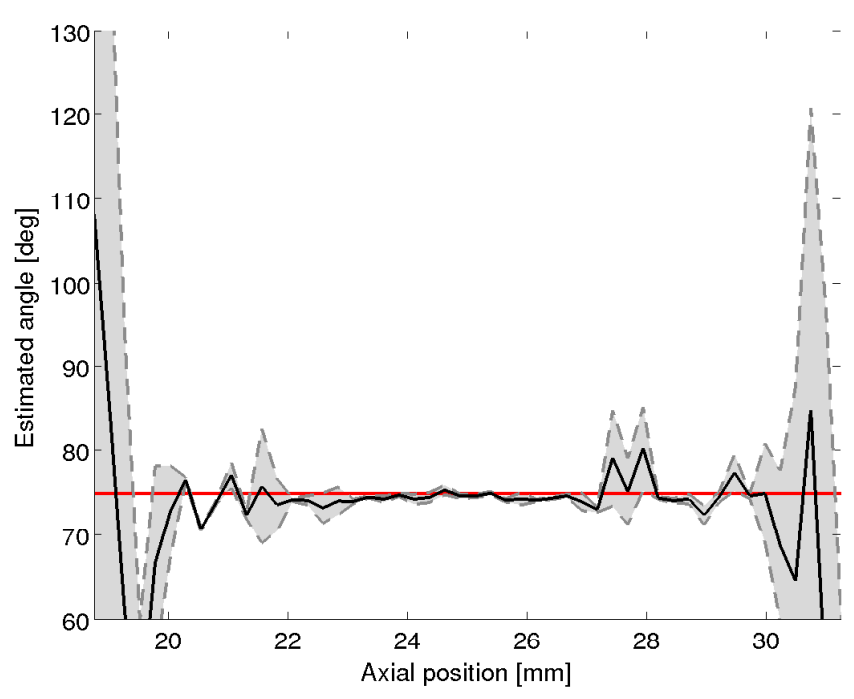

(d)

Figure 5: Simulated results for crossectional flow profile of the vessel with $v_{\text {peak }}=0.5 \mathrm{~m} / \mathrm{s}$ and $\theta=90^{\circ}$ for $k_{\text {tprf }}$ of 2 (left), and the vessel with $v_{\text {peak }}=0.05 \mathrm{~m} / \mathrm{s}$ and $\theta=75^{\circ}$ for $k_{\text {tprf }}$ of 6 (right). The top graphs show the estimated mean velocity profile and the standard deviation. The lower graphs show the bias and the standard deviation of the angle when radial lines are beamformed at $5^{\circ}$ steps. 


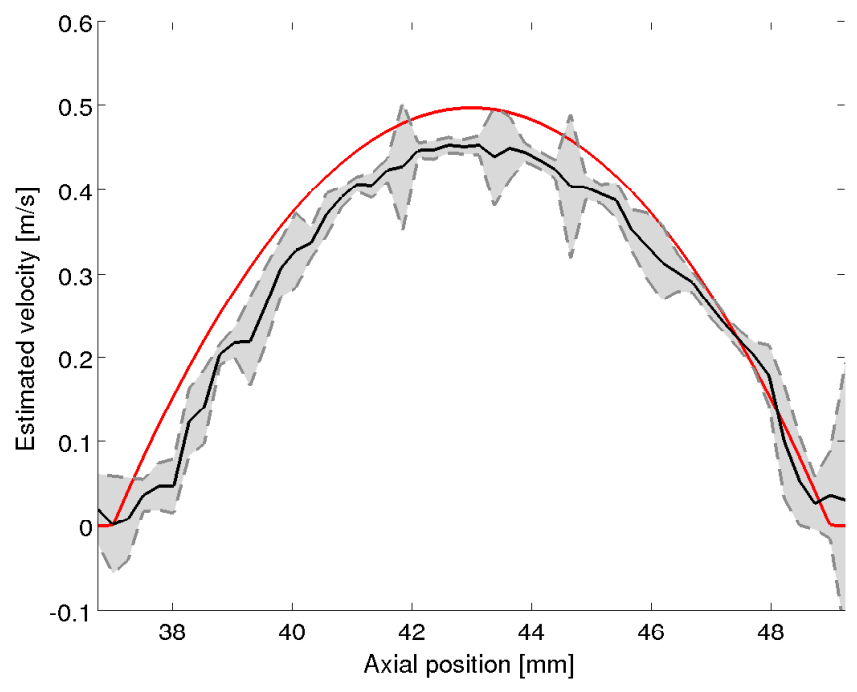

(a)

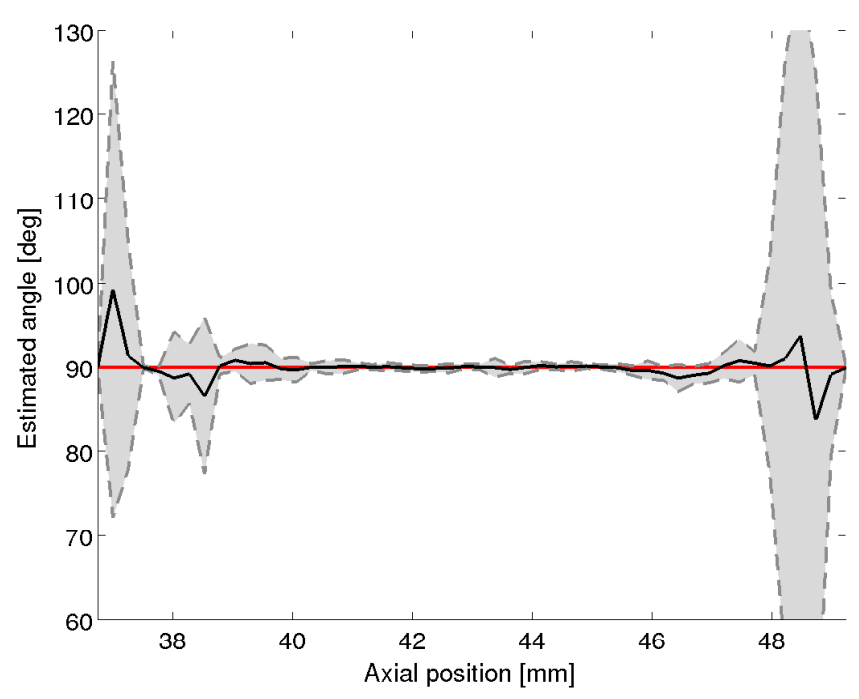

(c)

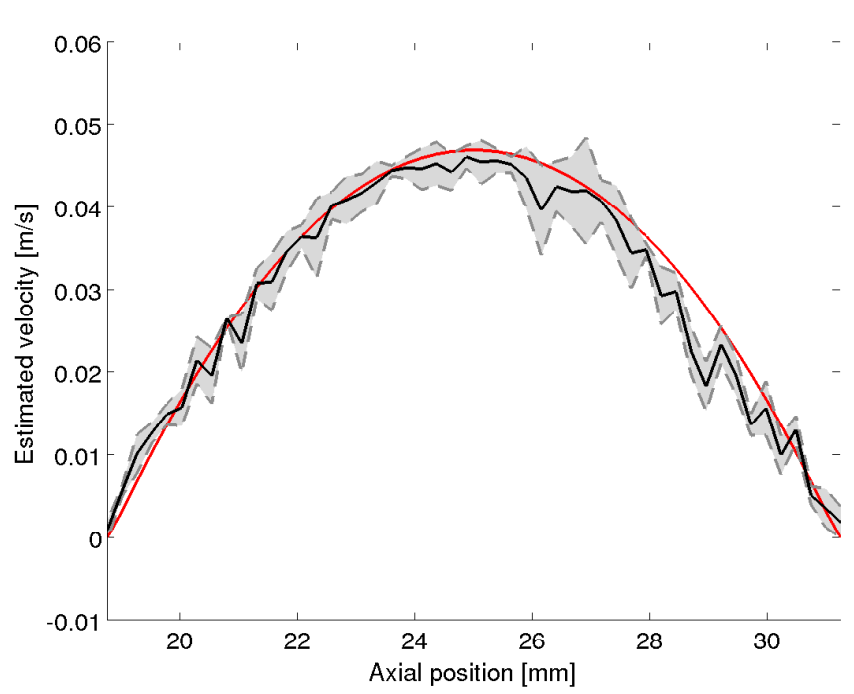

(b)

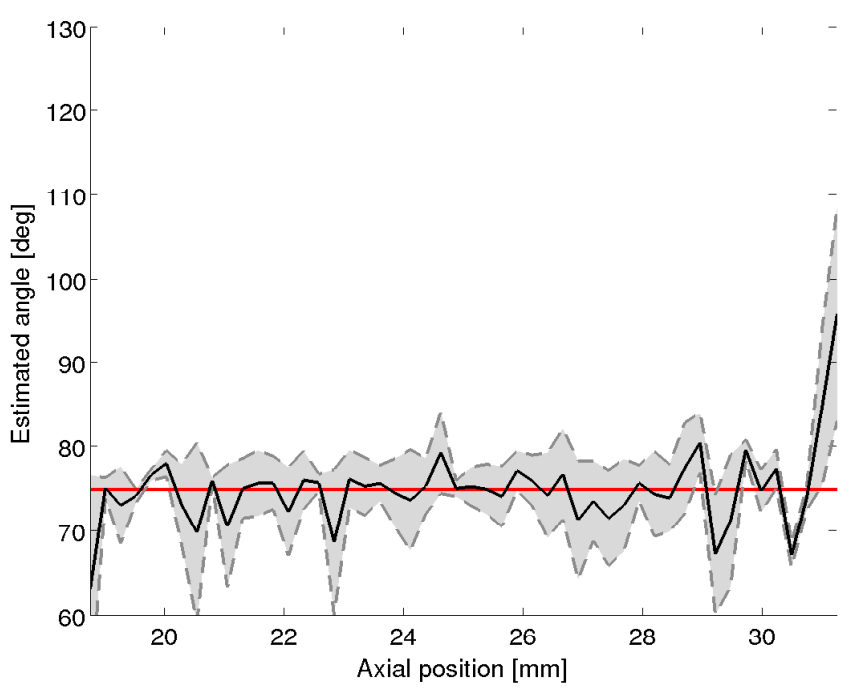

(d)

Figure 6: Measured results for crossectional flow profile of the vessel with $v_{\text {peak }}=0.5 \mathrm{~m} / \mathrm{s}$ and $\theta=90^{\circ}$ for $k_{\text {tprf }}$ of 2 (left), and the vessel with $v_{\text {peak }}=0.05 \mathrm{~m} / \mathrm{s}$ and $\theta=75^{\circ}$ for $k_{\text {tprf }}$ of 6 (right). The top graphs show the estimated mean velocity profile and the standard deviation. The lower graphs show the bias and the standard deviation of the angle when radial lines are beamformed at $5^{\circ}$ steps. 
Table 3: Bias and standard deviation of the estimates for the simulated vessels

\begin{tabular}{|c|c|c|c|c|c|c|c|c|}
\hline$v_{\text {peak }}$ & \multicolumn{4}{|c|}{$0.05 \mathrm{~m} / \mathrm{s}$} & \multicolumn{4}{|c|}{$0.5 \mathrm{~m} / \mathrm{s}$} \\
\hline \multirow[b]{2}{*}{$k_{t p r f}$} & \multicolumn{2}{|c|}{ rel. vel. } & \multicolumn{2}{|r|}{ angle } & \multicolumn{2}{|c|}{ rel. vel. } & \multicolumn{2}{|c|}{ angle } \\
\hline & bias & std. dev. & bias & std. dev. & bias & std. dev. & bias & std. dev. \\
\hline 1 & -8.97 & 35.91 & -0.45 & 17.80 & -4.87 & 1.77 & -0.05 & 2.00 \\
\hline 2 & -4.50 & 18.03 & -2.48 & 8.83 & -4.30 & 1.59 & -0.03 & 0.58 \\
\hline 4 & -2.10 & 5.95 & -0.80 & 3.24 & -8.24 & 19.51 & -0.07 & 2.53 \\
\hline 6 & 0.29 & 2.39 & -0.27 & 1.71 & -37.68 & 14.02 & 0.54 & 12.43 \\
\hline
\end{tabular}

\subsection{Experimental results}

The measurements are setup to match the geometry of the simulated vessels. However, due to the limitation that the flow rig only contains one mimicking vessel, the acquisitions for the two vessels are made independently. Fig. 6 show the estimated velocity profiles from the measurements. This is comparable with the simulation results. The estimated vector velocities for the two vessels, $v_{\text {peak }}=0.5$ and $0.05 \mathrm{~m} / \mathrm{s}$, have a relative bias for the magnitude of $-7.37 \%$ and $-3.19 \%$ with a relative standard deviation of $6.12 \%$ and $5.75 \%$, and the angle has a bias of $-0.09^{\circ}$ and $-0.38^{\circ}$, with a standard deviation of $5.65^{\circ}$ and $4.24^{\circ}$, respectively.

\section{DISCUSSION}

The fact that SA provides continuous data of the scatterer displacement, enables the synthesis of lower pulse repetition frequencies which allows the detection of lower velocities with the same dataset. Figure 4 (b), shows the result of applying different synthesized pulse repetition frequencies, represented as the change of $k_{t p r f}$. From this figure, it can be seen that the range of detectable velocity is shifted towards lower velocities. Since the same data set is used, the $k_{t p r f}$ can be selected independently for any estimation point, removing the lower limit on the velocity estimation. The selection of the appropriate $k_{t p r f}$ is a topic of further research. When measuring lower velocities vessels, the movement of the tissue can be on the same order of magnitude as the movement of the blood, lowering the performance of the echo-canceling filter and yielding erroneous blood velocity estimates. This challenge also requires further study.

Another advantage is that vector velocities can be estimated for every point independently without previous knowledge of the direction of the flow, allowing the detection of complex flows. Fig. 4 (a), shows the detection of the correct angle is practically independent from the magnitude of the velocity, and shows that the bias and standard deviations for both angle and magnitude are kept low for a range of velocities, Fig. 4 (c,d).

However, there is an issue for higher $\left(k_{t p r f}>4\right)$ where the angle estimates start failing more frequently for true flow directions lower than $30^{\circ}$. This can be attributed to the gradient phantom used in the study, as the phantom contains a large range of velocities in a small area. The faster moving scatterers in the vicinity can influence a higher decorrelation in the lower velocity scatterers, hence giving a lower maximum in the crosscorrelation and an erroneous angle detection. This primary seen in angles lower than $30^{\circ}$, since the point spread function is asymmetric. It is important to mention that Couette flow is not seen in a clinical environment, and it therefore remains to be seen if this effect has any influence in a real environment.

Finally, it should be noted that the method can accurately estimate the vector velocities for simulations and measurements, as shown in Fig. 5 and Fig. 6. Both show agreement with the true velocity profile with low standard deviations and bias. The simulations give better estimates since it is a noise free environment.In a measurement setup there are several noise sources lowering the signal-to-noise ratio which leads to higher standard deviation.

Additionally, the method can reduce the operator dependence of flow imaging since it can select the appropriate $k_{t p r f}$ for every estimation point. It also enables the possibility of new applications, such as pressure gradients, perfusion imaging. 


\section{CONCLUSION}

The performance of synthetic aperture, directional beamforming vector velocity estimator has been presented. It has been shown that the estimates can be done without previous knowledge of the direction of the flow, and with an extended range of detectable velocities it will be possible to accurately estimate velocities across the entire cardiac circle.

\section{REFERENCES}

1. C. Kasai, K. Namekawa, A. Koyano, and R. Omoto, "Real-Time Two-Dimensional Blood Flow Imaging using an Autocorrelation Technique," IEEE Trans. Son. Ultrason. 32, pp. 458-463, 1985.

2. D. Dotti, E. Gatti, V. Svelto, A. Uggè, and P. Vidali, "Blood flow measurements by ultrasound correlation techniques," Energia Nucleare 23, pp. 571-575, 1976.

3. S. I. Nikolov and J. A. Jensen, "Velocity estimation using synthetic aperture imaging," in Proc. IEEE Ultrason. Symp., pp. 1409-1412, 2001.

4. J. A. Jensen and S. I. Nikolov, "Transverse flow imaging using synthetic aperture directional beamforming," in Proc. IEEE Ultrason. Symp., pp. 1488-1492, 2002.

5. S. I. Nikolov and J. A. Jensen, "In-vivo Synthetic Aperture Flow Imaging in Medical Ultrasound," IEEE Trans. Ultrason., Ferroelec., Freq. Contr. 50(7), pp. 848-856, 2003.

6. J. A. Jensen and S. I. Nikolov, "A method for real-time three-dimensional vector velocity imaging," in Proc. IEEE Ultrason. Symp., pp. 1582-1585, 2003.

7. M. B. Stuart, B. G. Tomov, M. J. Pihl, and J. A. Jensen, "High frame rate synthetic aperture duplex imaging," in Proc. IEEE Ultrason. Symp., July 2013.

8. J. A. Jensen, "Velocity vector estimation in synthetic aperture flow and B-mode imaging," in IEEE International Symposium on Biomedical imaging from nano to macro, pp. 32-35, 2004.

9. J. A. Jensen, "Directional velocity estimation using focusing along the flow direction: I: Theory and simulation," IEEE Trans. Ultrason., Ferroelec., Freq. Contr. 50, pp. 857-872, 2003.

10. J. A. Jensen and R. Bjerngaard, "Directional velocity estimation using focusing along the flow direction: II: Experimental investigation," IEEE Trans. Ultrason., Ferroelec., Freq. Contr. 50, pp. 873-880, 2003.

11. S. I. Nikolov, K. Gammelmark, and J. A. Jensen, "Recursive ultrasound imaging," in Proc. IEEE Ultrason. Symp., 2, pp. 1621-1625, 1999.

12. J. A. Jensen and S. I. Nikolov, "Directional synthetic aperture flow imaging," IEEE Trans. Ultrason., Ferroelec., Freq. Contr. 51, pp. 1107-1118, 2004.

13. S. G. Foster, A pulsed ultrasonic flowmeter employing time domain methods. PhD thesis, Dept. Elec. Eng., University of Illinois, Urbana, Ill., 1985.

14. J. A. Jensen, Estimation of Blood Velocities Using Ultrasound: A Signal Processing Approach, Cambridge University Press, New York, 1996.

15. J. A. Jensen, "Field: A program for simulating ultrasound systems," Med. Biol. Eng. Comp. 10th NordicBaltic Conference on Biomedical Imaging, Vol. 4, Supplement 1, Part 1, pp. 351-353, 1996.

16. J. A. Jensen and N. B. Svendsen, "Calculation of Pressure Fields from Arbitrarily Shaped, Apodized, and Excited Ultrasound Transducers," IEEE Trans. Ultrason., Ferroelec., Freq. Contr. 39, pp. 262-267, 1992.

17. J. A. Jensen, O. Holm, L. J. Jensen, H. Bendsen, S. I. Nikolov, B. G. Tomov, P. Munk, M. Hansen, K. Salomonsen, J. Hansen, K. Gormsen, H. M. Pedersen, and K. L. Gammelmark, "Ultrasound Research Scanner for Real-time Synthetic Aperture Image Acquisition," IEEE Trans. Ultrason., Ferroelec., Freq. Contr. 52 (5), pp. 881-891, May 2005.

18. K. V. Ramnarine, D. K. Nassiri, P. R. Hoskins, and J. Lubbers, "Validation of a new blood mimicking fluid for use in Doppler flow test objects," Ultrasound Med. Biol. 24, pp. 451-459, 1998.

19. J. M. Hansen, M. C. Hemmsen, and J. A. Jensen, "An object-oriented multi-threaded software beamformation toolbox," in Proc. SPIE Med. Imag., 7968, pp. 79680Y 1-9, March 2011. 